\title{
The Use of Scanning Electron Microscopy and Microanalysis to Classify Dentin
}

\author{
E.T. Coutinho, J.R.M. d'Almeida and S. Paciornik
}

Materials Science and Metallurgy Department, Pontifícia Universidade Católica do Rio de Janeiro, Rua Marquês de São Vicente, 225 - Gávea - 22453-900, Rio de Janeiro, RJ, Brazil.

One of the most usual procedures in dentistry is the substitution of old restorations. In many instances, however, "old" one means a restoration with just 3 years of use [1]. Moreover, only one third of all restorations derive from a new dental lesion. The main cause of restoration substitution is their failure, and $95 \%$ of all failure events are related to the fracture of the interface between tooth and restoration [2].

Promoting a better adhesion between the tooth and the restoration is, therefore, a relevant goal. In fact, the usual procedure of acid cleaning is aimed at enhancing adhesion. Since dentin is, in many instances, the largest portion of the substrate, the analysis of dentin for restoration adhesion is, nowadays, the focus of many works [3]. Adhesion is, however, not a simple matter and in order to completely understand the adhesion behavior of a substrate/adhesive pair one has to determine the mechanical behavior of both and also fully characterize the surface features of the substrate.

In this work the morphological aspects of human dentin from unerupted third molars were characterized by scanning electron microscopy (SEM) and their elemental constitution was determined by energy dispersive X-rays (EDS). The objective of the work was to identify regions of common characteristic features on different teeth, and along a single tooth. A systematic classification of dentin is considered essential to understand why some restorations fail much earlier than other performed under the same carefully controlled clinical conditions.

The analysis was performed on the occlusal surface of teeth. The techniques of preparation of these surfaces are reported elsewhere [4]. The number of dentine tubules per unit area, their size, shape and distribution were automatically measured using a specially developed image analysis procedure. Fig.1 shows that the microstructure of dentin has a strong variation as a function of the region analyzed. At the central region of the teeth, under the main groove, medium to large regularly spaced tubules of circular shape are observed, Fig.1a. The number of tubules per unit area of this region is small, thus increasing the inter-tubular dentin fraction. The regions under the cusps and near the pulp horns are characterized by large circular tubules, Fig.1b. In these regions the number of tubules is very high. The main fraction of dentin, between the grooves and the cusps, is characterized by a large number of small to medium, regularly spaced, tubules, Fig.1c. In this region the projected shapes of the tubules vary from circular to elliptical. Finally, periphery regions near the dentinenamel junction show a small number of small tubules, irregularly distributed and elliptically shaped, Fig. 1d. This region is by far the less homogeneous, and wide differences are found in respect to the shape, size and distribution of the tubules.

Fig. 2 shows a typical spectrum obtained by EDS. Differently from the SEM, the results do not show significant variations from one position to another along the same teeth. The only elements present are $\mathrm{Ca}$ and $\mathrm{P}$ (the Au peak came from the conductive sputtered layer). This result shows that any relevant difference on the adhesion behavior of restorations should be due primarily to the dentin region participating on the adhesion pair, and not to the constitution of the dentin itself. 
From the results obtained, each occlusal surface was classified in respect to the morphology of the dentin, as shown in Fig.3 for three of the teeth studied. One can see that the relative fraction and position of each region vary from tooth to tooth. Generalization of the hardness behavior of the analyzed teeth was done using this classification [5]. It is expected that the tensile values from different dentin-restoration regions can be better understood after systematically mapping the dentin surface [6].

\section{References:}

[1] Mjor, I.A., Acta Odontologica Scandinavica, 55, 58-63, 1997.

[2] Duke, E.S., Compendium of Continuing Education in Dentistry, 20, 1010-1012, 1999.

[3] Nakabayashi, N., Pashley, D.H., Hybridization of dental hard tissues, Tokyo:Quintessence, 1998.

[4] Coutinho, E.T., d'Almeida, J.R.M., Paciornik, S. submitted, 2003.

[5] Coutinho, E.T., d'Almeida, J.R.M., Paciornik, S. in preparation, 2003.

[6] This work was partially funded by the Brazilian agency $\mathrm{CNPq}$.
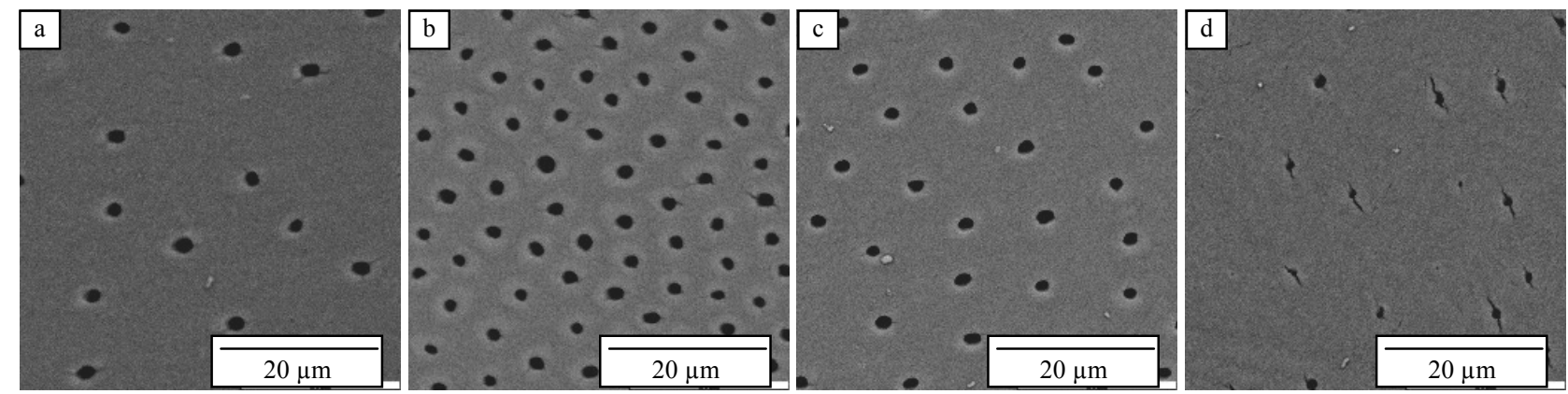

Fig. 1 - Variation of the size, shape and distribution of the tubules in the dentin.

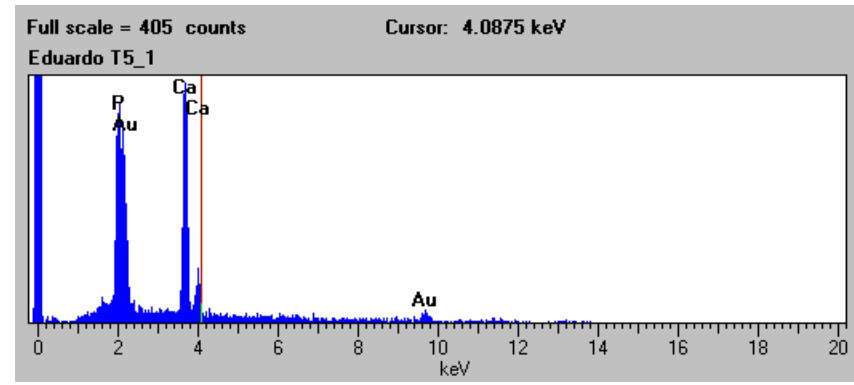

Fig.2 - Typical EDS spectra obtained at all positions for all analyzed teeth.
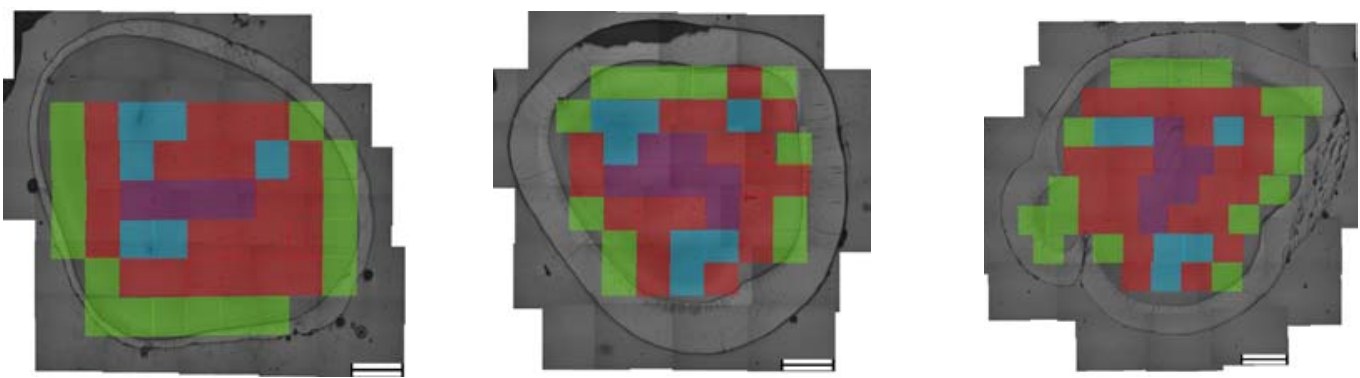

Fig.3 - Classification of the dentin as a function of its position and morphology. In lilac are regions with the characteristics of Fig. 1a; in blue from Fig.1b; in red from Fig.1c, and in green from Fig.1d. 